\title{
Direct admission to a surgical service reduces hospital length of stay for patients with biliary disease
}

\author{
Gregory S. Corwin, Rebecca Reif, Kevin W. Sexton* \\ Department of Surgery, Division of Trauma and Acute Care Surgery, University of Arkansas for Medical Sciences, Arkansas, \\ United States
}

Received: February 2, 2019

Accepted: March 3, 2019

Online Published: April 15, 2019

DOI: $10.5430 /$ jha.v8n3p1

URL: https://doi.org/10.5430/jha.v8n3p1

\begin{abstract}
Background: Biliary tract disease is a common condition often necessitating surgical intervention. It has been suggested that categorically admitting these patients to a surgical service rather than a medical service may improve patient outcomes. Our objective was to assess the impact of a protocol change that mandated preferentially admitting patients with biliary disease to a surgical service.

Methods: This is a retrospective observational study of patients presenting with biliary disease to a single institution before and after a protocol change that mandated admitting these patients directly to a surgical service. A generalized linear model was conducted to analyze the effect of practice change on length of stay, which was primary studied outcome.

Results: A total of 3,389 patients were included in the study ( $n=1,866$ for pre, and $n=1,523$ for post). There was no difference in hospital length of stay between pre and post groups for non-operative patients (1.9 days \pm 4.3 versus 1.9 days $\pm 5.2, p=.972$ ). However, for operative patients, length of stay was shorter for the post group (4.1 days \pm 6.1 vs. 6.3 days $\pm 14.0, p=.066$ ). The generalized linear model found that operative patients had an increased probability of having a longer length of stay (coefficient, $0.21 ; 95 \%$ CI, $0.14,0.29 ; p<.001)$.

Conclusion: Admission of patients with biliary disease to a surgical service rather than a medical service is associated with shorter length of stay for patients who undergo an operative intervention. An approach of admitting all patients presenting with biliary disease to a surgical service has the potential to significantly reduce hospital costs. Our study supports primary responsibility for surgeons in the care of patients with potentially operative conditions.
\end{abstract}

Key Words: Biliary disease, Surgical admission, Length of stay, Surgery

\section{INTRODUCTION}

Biliary tract disease is a common surgical condition with a major health burden that has increased more than $20 \%$ over the last 3 decades. ${ }^{[1]}$ It has been common practice for patients with conditions that have traditionally been viewed as surgical to initially be admitted to medical services; this occurs particularly with conditions for which the management is often non-operative. ${ }^{[2,3]}$ The admission decisions can be driven by comorbidities, institutional practice patterns, or other factors. Over recent years, this practice has increasingly come under question with the publication of studies demonstrating the importance of ongoing surgical involvement with patients having potentially surgical conditions, as well as improvement in outcomes associated with admission of these patients to surgical services as opposed to medical services. ${ }^{[2-5]}$ Further reinforcing the argument for

\footnotetext{
* Correspondence: Kevin W. Sexton, MD; Email: kevin.sexton@uams.edu; Address: Department of Surgery, Division of Trauma and Acute Care Surgery University of Arkansas for Medical Sciences (UAMS), Little Rock, AR 72205, United States.
} 
more direct responsibility of surgeons in the care of patients with potentially operative conditions is the maturation over recent years of Acute Care Surgery (ACS) as an integral part of Trauma Surgery. ${ }^{[6-8]}$

Surgery of the biliary tract is among of the most common surgeries, with over 400,000 cholecystectomies performed in 2012. ${ }^{[9]}$ However, biliary disease including acute cholecystitis, common bile duct stones, and gallstone pancreatitis are conditions often admitted to medical services. There have been few studies that have looked at the impact of admission service for patients with biliary disease. Several studies have examined the effect of admission service on outcomes for patients with either gallstone pancreatitis ${ }^{[10,11]}$ or acute cholecystitis, ${ }^{[5]}$ finding less time to surgery and/or shorter length of stay associated with admission to a surgery service in contrast to admission to a medical service. Similarly, establishing an ACS model of care was shown to decrease hospital length of stay and complication rates in patients with acute cholecystitis ${ }^{[12,13]}$ or gall bladder surgery. ${ }^{[14]}$ In contrast, others have not found long-term differences in outcomes between patents with gallstone pancreatitis admitted to either a medical or surgical team or any significant improvement in outcomes in patients with biliary disease with implementation of an ACS program. ${ }^{[15,16]}$

Historically our institution has not had an established policy as to whether a patient presenting with biliary disease was admitted to a medical service or admitted to a surgical service. In 2016, we established the practice of preferentially admitting all patients with biliary disease to the surgery service. The objective of the current study was to examine whether this mandated admission to a surgical service as compared to our prior practice model shortened length of stay for patients presenting with biliary disease.

\section{MethodS}

\subsection{Study setting}

The University of Arkansas for Medical Sciences (UAMS) is a tertiary care university teaching hospital with a total of 405 adult patient beds. UAMS is an American College of Surgeons Verified Level 1 Trauma Center, and the Acute Care Surgery Service, which includes an in-house attending surgeon, is available 24 hours a day. Data for the study were provided by the Arkansas Clinical Data Repository (AR-CDR) maintained by the Department of Biomedical Informatics in the College of Medicine at the UAMS. The AR-CDR is approved to operate as an enterprise data resource to support research across UAMS. Data in the AR-CDR comes from UAMS Electronic Medical Record (EMR), tumor registry, billing, and cancer genomic data, and comprises encounters since 05/01/2014.

\subsection{Study design}

This was a retrospective observational study, where patients admitted between May 2014 and December 2016 were included. Prior to August 2015, there were no set criteria for whether a patient presenting to UAMS with biliary disease was admitted to a medical service or to a surgical service (with surgical consult if requested). Beginning in August 2015 , a practice change was initiated to preferentially admit all patients presenting with biliary disease to a surgical service with a surgeon as the attending physician. Patients before August 2015 were classified as "pre" protocol change, and patients during and after August 2015 were included in the "post" protocol change group. This study was approved by the institutional review board of UAMS.

\subsection{Patients}

The initial data set consisted of 12,945 patients with a diagnosis of biliary disease. We focused on the initial encounters of adult patients diagnosed with cholecystitis or bile duct blockages. Based on ICD-9 and ICD-10 diagnosis codes, patients were grouped into three diagnostic groups: cholecystitis, choledocholithiasis, and symptomatic cholelithiasis (see Table 1). The pre-group included all visits between May 2014 and July 2015 while the post-group included admissions between August 2015 and December 2016.

Patient admissions were classified either being inpatient, observation, or emergency. If, during an admission a patient underwent a surgical procedure the patient was considered operative patient, otherwise they were considered non-operative. Based on Current Procedural Terminology (CPT) codes, surgical procedures were classified into four categories: common bile duct explorations, percutaneous cholecystectomy drains, endoscopic retrograde cholangiopancreatographies, and laparoscopic cholecystectomies (see Table 2).

Demographic and outcome data were obtained from the Arkansas Clinical Data Repository (AR-CDR) maintained by the Department of Biomedical Informatics in the College of Medicine at UAMS. The Elixhauser Comorbidity Index was calculated according to the readmission coefficients used by Moore et al. ${ }^{[17]}$

\subsection{Primary outcome}

The primary outcome was to determine hospital length of stay (days) before and after the practice change was implemented.

\subsection{Statistical analysis}

Descriptive statistics were used to summarize the preintervention and post-intervention data. Bivariate analysis was used to test for the difference between the subgroup 
means for patients' pre-intervention and post-intervention by operative and non-operative status. The differences between the group means on each measure were analyzed for direction and statistical significance using $t$-tests for continuous variables and chi-square tests for categorical variables. Statistical significance was set at $\alpha=0.05$ for all analyses. Controlling for patient and clinical characteristics, a generalized linear model was conducted to analyze the effect of practice change on length of stay. The analysis was conducted using Stata. ${ }^{[15,18]}$

Table 1. Diagnostic Groups by ICD-9 and ICD-10 Codes

\begin{tabular}{|c|c|c|}
\hline Diagnostic Group & ICD-9 & ICD-10 \\
\hline \multirow[t]{12}{*}{ Cholecystitis } & 574 & K80.00 \\
\hline & 574.01 & K80.01 \\
\hline & 574.1 & K80.10 \\
\hline & 574.11 & K80.11 \\
\hline & 574.61 & K80.12 \\
\hline & 574.70 & K80.13 \\
\hline & 574.71 & K80.18 \\
\hline & 575 & K80.19 \\
\hline & 575.1 & K80.60 \\
\hline & 575.11 & K80.62 \\
\hline & 575.12 & K80.64 \\
\hline & 575.20 & \\
\hline \multirow[t]{22}{*}{ Choledocholithiasis } & 574.3 & K80.21 \\
\hline & 574.31 & K80.30 \\
\hline & 574.4 & K80.31 \\
\hline & 574.41 & K80.32 \\
\hline & 574.5 & K80.40 \\
\hline & 574.51 & K80.41 \\
\hline & 574.9 & K80.42 \\
\hline & 574.91 & K80.43 \\
\hline & 576.1 & K80.44 \\
\hline & 576.20 & K80.45 \\
\hline & & K80.46 \\
\hline & & K80.47 \\
\hline & & K80.50 \\
\hline & & K80.51 \\
\hline & & K80.63 \\
\hline & & K80.66 \\
\hline & & K80.67 \\
\hline & & K80.70 \\
\hline & & K80.71 \\
\hline & & K80.81 \\
\hline & & K83.0 \\
\hline & & K83.1 \\
\hline Symptomatic & 574.20 & K80.20 \\
\hline \multirow[t]{2}{*}{ Cholelithiasis } & 574.21 & K80.21 \\
\hline & & K80.80 \\
\hline
\end{tabular}

Table 2. Procedure Breakdown by CPT Codes

\begin{tabular}{ll}
\hline Procedure & CPT codes \\
\hline Common Bile Duct & $47420,47480,47564,47600$, \\
Exploration & $47605,47610,47612$ \\
& $47490,47500,47505,47510$, \\
Percutaneous & $47511,47525,47530,47531$, \\
Cholecystostomy Drain & $47532,47533,47534,47535$, \\
& 47536 \\
Endoscopic Retrograde & $47538,47542,47543,47544$, \\
Cholangiopancreatography & $47630,47550,47552,47553$, \\
& $47554,47555,47556$ \\
Laparoscopic & 47562,47563 \\
Cholecystectomy & \\
\hline
\end{tabular}

\section{REsults}

\subsection{Patient demographics}

A total of 3,389 patients were included in the study; 1,866 pre-practice change and 1,523 post-practice change (see Figure 1). Patients who died during their hospital stay were excluded from the analysis. Table 3 summarizes the descriptive statistics of the pre-intervention and post-intervention patient populations. There were some differences noted between the two patient populations in patient type, diagnostic group, and surgical procedures. There was more acute cholecystitis and less asymptomatic choledocholithiasis in the pre-group as well as more laparoscopic cholecystectomies performed. Similarly, there were significant differences in the pre-intervention and post-intervention patient populations with respect to patient types and diagnostic groups for both operative and non-operative patients (see Tables 4 and 5). However, there were no differences between the preintervention and post-intervention patient populations with respect to the surgical procedures that were performed (see Table 5).

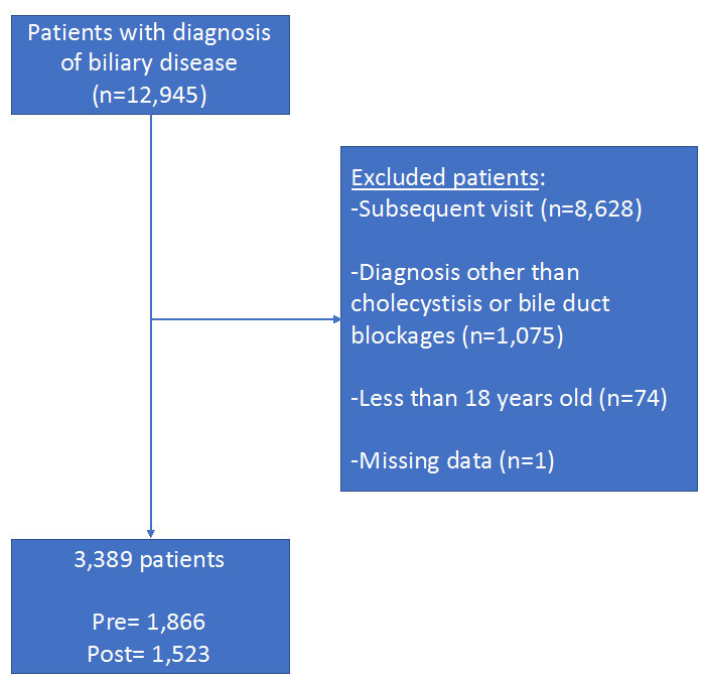

Figure 1. Patient inclusion flow diagram 
Table 3. Study population characteristics $(\mathrm{n}=3,389)$

\begin{tabular}{lll}
\hline Characteristic & $\begin{array}{l}\text { Pre } \\
(\mathbf{n}=\mathbf{1 , 8 6 6})\end{array}$ & $\begin{array}{l}\text { Post } \\
\mathbf{( n = 1 , 5 2 3 )}\end{array}$ \\
\hline Age, y & $54.5 \pm 17.0$ & $54.8 \pm 17.7$ \\
18-40, n (\%) & $440(23.6)$ & $383(25.2)$ \\
41-64, n (\%) & $860(46.1)$ & $643(42.2)$ \\
64-89, n (\%) & $566(30.3)$ & $497(32.6)$ \\
Male Gender, n (\%) & $740(39.7)$ & $592(38.9)$ \\
Readmit, n (\%) & $31(1.71)$ & $23(1.51)$ \\
Length of Stay & $2.7 \pm 7.1$ & $2.1 \pm 5.2$ \\
Patient Type & & \\
$\quad$ Emergency, n (\%) & $232(12.4)$ & $235(15.4)$ \\
$\quad$ Inpatient, n (\%) & $549(29.4)$ & $340(22.3)$ \\
$\quad$ Observation, n (\%) & $1,085(58.2)$ & $948(62.3)$ \\
Diagnostic Group & & \\
$\quad$ Cholecystitis, n (\%) & $234(12.5)$ & $75(4.9)$ \\
$\quad$ Choledocholithiasis, n (\%) & $346(18.5)$ & $454(29.8)$ \\
$\quad$ Symptomatic Choledocholithiasis, n (\%) & $1,286(68.9)$ & $994(65.3)$ \\
Procedure & $308(16.5)$ & $150(9.9)$ \\
$\quad$ Common Bile Duct Exploration, n (\%) & $27(1.5)$ & $16(1.1)$ \\
Endoscopic Retrograde Cholangiopancreatography, n (\%) & $29(1.6)$ & $15(1.0)$ \\
$\quad$ Laparoscopic Cholecystectomy, n (\%) & $190(10.2)$ & $88(5.8)$ \\
$\quad$ Percutaneous Cholecystostomy Drain, n (\%) & $62(3.32)$ & $31(2.0)$ \\
Elixhauser Comorbidity Index & $29[10-54]$ & $25[8-46]$ \\
\hline
\end{tabular}

Table 4. Characteristics of non-operative patients

\begin{tabular}{llll}
\hline Characteristic & $\begin{array}{l}\text { Pre } \\
(\mathbf{n = 1 , 5 5 8 )}\end{array}$ & $\begin{array}{l}\text { Post } \\
(\mathbf{n = 1 , 3 7 3 )}\end{array}$ & p-value \\
\hline Age, y & $55.4 \pm 16.9$ & $55.3 \pm 17.6$ & .904 \\
18-40, n (\%) & $339(21.8)$ & $331(24.1)$ & \\
41-64, n (\%) & $721(46.3)$ & $579(42.2)$ & .073 \\
64-89, n (\%) & $498(32.0)$ & $463(33.7)$ & \\
Male Gender, n (\%) & $625(40.1)$ & $537(39.1)$ & .579 \\
Readmit, n (\%) & $13(0.83)$ & $15(1.09)$ & .473 \\
Length of Stay & $1.9 \pm 4.3$ & $1.9 \pm 5.2$ & .972 \\
Patient Type & & & \\
$\quad$ Emergency & $195(12.5)$ & $221(16.1)$ & \\
Inpatient & $387(24.84)$ & $277(20.2)$ & .001 \\
Outpatient & $976(62.6)$ & $875(63.7)$ & \\
Diagnostic Group & & & \\
Cholecystitis & $125(8.0)$ & $48(3.5)$ & \\
Choledocholithiasis & $248(15.9)$ & $398(29.0)$ & $<.001$ \\
$\begin{array}{l}\text { Symptomatic } \\
\text { Choledocholithiasis }\end{array}$ & $1185(76.1)$ & $927(67.52)$ & \\
$\begin{array}{l}\text { Elixhauser } \\
\text { Comorbidity Index }\end{array}$ & $31[11-54]$ & $26[8-47]$ & $<.001$ \\
\hline
\end{tabular}

\subsection{Hospital length of stay}

There was no difference in hospital length of stay between pre-intervention and post-intervention groups for nonoperative patients (1.9 days \pm 4.3 versus 1.9 days $\pm 5.2, p=$ .972). In contrast, for operative patients, length of stay was shorter for the post patient population as compared to the pre-intervention patient population (4.1 days \pm 6.1 versus 6.3 days $\pm 14.0, p=.066$ ). The generalized linear model (controlling for protocol, operative status, age, gender, patient type, procedure, diagnosis, and Elixhauser Comorbidity Index) found that operative patients had an increased probability of having a longer length of stay compared to nonoperative patients (coefficient, 0.21; 95\% CI, 0.14, 0.29; $p<$ .001) (see Table 6). On the other hand, the generalized linear model for length of stay in operative patients (controlling for protocol, age, gender, patient type, procedure, diagnosis, and Elixhauser Comorbidity Index) found that post-intervention patients had a decreased probability of having a longer length of stay as compared to pre-intervention patients (coefficient, $-0.26 ; 95 \%$ CI, $-0.35,-0.17 ; p<.001$ ) (see Table 7). 
Table 5. Characteristics of operative patients

\begin{tabular}{|c|c|c|c|}
\hline Characteristic & $\begin{array}{l}\text { Pre } \\
(n=308)\end{array}$ & $\begin{array}{l}\text { Post } \\
(n=150)\end{array}$ & $p$-value \\
\hline Age, $y$ & $50.2 \pm 16.5$ & $50.3 \pm 18.2$ & .952 \\
\hline 18-40, n (\%) & $101(32.8)$ & $52(34.7)$ & \\
\hline 41-64, n (\%) & $139(45.1)$ & $64(42.7)$ & .878 \\
\hline 64-89, n (\%) & $68(22.1)$ & $34(22.7)$ & \\
\hline Male Gender, n (\%) & 115 (37.3) & 55 (36.7) & .889 \\
\hline Readmit, n (\%) & 19 (6.17) & $8(5.33)$ & .722 \\
\hline Length of Stay & $6.3 \pm 14.0$ & $4.1 \pm 6.1$ & .066 \\
\hline \multicolumn{4}{|l|}{ Patient Type } \\
\hline Emergency & $37(12.0)$ & $14(9.3)$ & \multirow{3}{*}{.024} \\
\hline Inpatient & $162(52.6)$ & $63(42.0)$ & \\
\hline Outpatient & $109(35.4)$ & 73 (48.7) & \\
\hline \multicolumn{4}{|l|}{ Diagnostic Group } \\
\hline Cholecystitis & 109 (35.4) & $27(18.0)$ & \multirow{3}{*}{.001} \\
\hline Choledocholithiasis & $98(31.8)$ & $56(37.3)$ & \\
\hline $\begin{array}{l}\text { Symptomatic } \\
\text { Choledocholithiasis }\end{array}$ & $101(32.8)$ & $67(44.7)$ & \\
\hline \multicolumn{4}{|l|}{ Procedure } \\
\hline $\begin{array}{l}\text { Common Bile Duct } \\
\text { Exploration, n (\%) } \\
\text { Endoscopic Retrograde }\end{array}$ & $27(8.8)$ & 16 (10.7) & \multirow{5}{*}{.899} \\
\hline $\begin{array}{l}\text { Cholangiopancreato- } \\
\text { graphy, n (\%) }\end{array}$ & $29(9.4)$ & $15(10.0)$ & \\
\hline $\begin{array}{l}\text { Laparoscopic } \\
\text { Cholecystectomy, n (\%) } \\
\text { Percutaneous }\end{array}$ & $190(61.7)$ & $88(58.7)$ & \\
\hline $\begin{array}{l}\text { Cholecystostomy Drain, } \\
\text { n (\%) }\end{array}$ & $62(20.1)$ & $31(20.7)$ & \\
\hline $\begin{array}{l}\text { Elixhauser Comorbidity } \\
\text { Index }\end{array}$ & 22 [8-43] & 21.5 [4-42] & \\
\hline
\end{tabular}

Table 6. General linear model for length of stay

\begin{tabular}{llll}
\hline Variable & Coefficient & 95\% CI & $\boldsymbol{p}$-value \\
\hline Post protocol & -0.00 & $(-0.05,0.04)$ & .879 \\
Operative & 0.21 & $(0.14,0.29)$ & $<.001$ \\
Age: $41-64$ & -0.21 & $(-0.27,-0.16)$ & $<.001$ \\
Age: 65-89 & -0.15 & $(-0.23,-0.11)$ & $<.001$ \\
Male & -0.01 & $(-0.04,0.05)$ & .772 \\
Pttype: Inpatient & 1.49 & $(1.44,1.55)$ & $<.001$ \\
Pttype: Emergency & 0.05 & $(-0.06,0.15)$ & .372 \\
Common Bile Duct & 0.53 & $(0.43,0.63)$ & $<.001$ \\
Exploration & & & \\
Percutaneous & & $(0.48,0.66)$ & $<.001$ \\
Cholecystostomy & 0.57 & & \\
Drain & & & \\
$\begin{array}{l}\text { Endoscopic } \\
\text { Retrograde }\end{array}$ & & $(-0.16,0.07)$ & .425 \\
Cholangiopancreatogr & -0.04 & & \\
aphy & & $(0.10,0.25)$ & $<.001$ \\
Cholecystitis & 0.17 & $(0.18,0.29)$ & $<.001$ \\
$\begin{array}{l}\text { Choledocholithiasis } \\
\text { Elixhauser }\end{array}$ & 0.23 & $0.01,0.01)$ & $<.001$ \\
Comorbidity Index & 0.01 & & \\
\hline & & & \\
\hline
\end{tabular}

Table 7. General linear model for length of stay in operative patients

\begin{tabular}{llll}
\hline Variable & Coefficient & $\mathbf{9 5 \%}$ CI & $\boldsymbol{p}$-value \\
\hline Post protocol & -0.26 & $(-0.35,-0.17)$ & $<.001$ \\
Age: 41-64 & -0.16 & $(-0.26,-0.05)$ & .005 \\
Age: 65-89 & -0.09 & $(-0.20,0.03)$ & .150 \\
Male & 0.08 & $(-0.00,0.17)$ & .053 \\
Pttype: Inpatient & 2.09 & $(1.93,2.25)$ & $<.001$ \\
Pttype: Emergency & -0.01 & $(-0.32,0.30)$ & .962 \\
Common Bile Duct & 0.44 & $(0.34,0.53)$ & $<.001$ \\
Exploration & & & \\
$\begin{array}{l}\text { Percutaneous } \\
\text { Cholecystostomy }\end{array}$ & 0.56 & $(0.45,0.66)$ & $<.001$ \\
$\begin{array}{l}\text { Drain } \\
\text { Endoscopic }\end{array}$ & & & \\
$\begin{array}{l}\text { Retrograde } \\
\text { Cholangiopancreatog }\end{array}$ & 0.08 & $(-0.04,0.20)$ & .172 \\
raphy & & & \\
Cholecystitis & -0.38 & $(-0.52,-0.25)$ & $<.001$ \\
$\begin{array}{l}\text { Choledocholithiasis } \\
\text { Elixhauser }\end{array}$ & -0.53 & $(-0.68,-0.39)$ & $<.001$ \\
Comorbidity Index & 0.01 & $(0.01,0.01)$ & $<.001$ \\
\hline & & & \\
\hline
\end{tabular}

\section{Discussion}

This study is unique in that it is to date the largest study of the impact of admission protocol of patients with biliary disease to a surgical service, and includes patients who span the entire range of biliary disease; including acute cholecystitis, symptomatic gall stones, and asymptomatic gall stone disease. Our major finding is that patients with biliary disease, preferentially admitted to a surgical service and having an operative intervention, have a greater probability of having a shorter hospital length of stay. In fact, the length of stay for these operative patients was almost two days shorter than prior to the change in care admission process. On the other hand, for non-operative patients there was no change in length of stay.

There have been few prior studies looking at admission service and outcome for individuals presenting with biliary disease. Daniak et al., in a small retrospective study of patients who underwent laparoscopic cholecystectomy for acute cholecystitis, found that patients admitted to the surgical service had a shorter time to surgery, less surgical complications, and a shorter length of stay. ${ }^{[5]}$ Much of the delay in surgery was due to more diagnostic studies being performed. There have also been three retrospective studies looking at surgical admission for gall stone pancreatitis. ${ }^{[10,11,16]}$ Judkins et al. found patients admitted to the surgery service were more likely to have a cholecystectomy during that hospitalization, as well as fewer laboratory tests, antibiotics, and consultations. ${ }^{[10]}$ Similarly, Kulvatunyou et al. demonstrated that 
patients with mild gallstone pancreatitis admitted to the surgical service undergoing a cholecystectomy had a shortened time to surgery and hospital length of stay as compared to patients admitted to a medical service. ${ }^{[11]}$ On the other hand, LaFemia et al. in a long-term study of patients with gallstone pancreatitis reported that, while patients admitted to a surgical service were more likely to have a surgical procedure during the index hospitalization and fewer subsequent readmissions, in long-term follow-up there were no differences in total hospital days or cost for management associated with admitting team. ${ }^{[16]}$ The impact of admission service on time to surgery and length of stay is not unique to biliary disease. Similar findings have been reported in patients presenting with small bowel obstruction. ${ }^{[3,4]}$

We did not specifically look at time to surgery, however the fact that a higher likelihood for a shortened length of stay was only seen in operative patients would be consistent with prior studies showing shorter time to surgery impacting length of stay on surgical services. ${ }^{[16]}$ There were significantly fewer surgical procedures performed following the change in practice (10\% versus $17 \%$ ) which may reflect admission of fewer patients with acute cholecystitis and symptomatic stone disease in the post-protocol period. However, the types of surgical interventions performed in both time periods were comparable, with a majority of patients undergoing laparoscopic cholecystectomy, making it is less likely that the length of stay differences we observed were a result of differences in the characteristics of the populations between the two periods of time.

In addition to the change to admission service policy for biliary disease, we also have an ACS service which may have had some impact on outcome. An ACS model has been shown to decrease time to surgery and shorten length of stay for patients presenting to the Emergency Department with biliary disease and/or who required emergency cholecystectomy. ${ }^{[12-14]}$ On the other hand, in a study of hospitalized patients with biliary disease an ACS service had no significant impact on length of stay. ${ }^{[15]}$ In these studies, ACS served as a consultant service, as such ACS would be more likely to influence care in the Emergency Department or for medical admissions. In our study, if anything, ACS would have pos-

\section{REFERENCES}

[1] Everhart JE, Ruhl CE. Burden of digestive diseases in the United States part I: overall and upper gastrointestinal diseases. Gastroenterology. 2009; 136(2): 376-386. PMid:19124023. https://doi . org/10.1053/j.gastro.2008.12.015 itively influenced care in the period prior to policy change when more patients were admitted to medical services.

There are several limitations of the study. First, as a retrospective study there are the inherent limitations of any retrospective study in terms of data availability and limitations in establishing cause and effect. Second, given the before-after design we cannot rule out other changes in care that could have influenced our findings. Third, we do not know what proportion of patients were on the surgical service before the change admission policy or the proportion of patients still admitted to medical services after the admission policy change. Finally, there were several differences in the characteristics of the populations before and after the change. However, for the operative patients the relative proportions of the types of surgical interventions were comparable between the time periods. Similarly, the breakdown between emergency, inpatient, and observation patients were comparable between time periods.

In conclusion, preferential admission of patients with biliary disease to a surgical service rather than a medical service was associated with shorter length of stay for patients who undergo an operative intervention, and a comparable length of stay for non-operative patients. Given the frequency of biliary disease, an approach of admitting all patients presenting with biliary disease to a surgical service has the potential to significantly reduce hospital costs. Our study supports a position for more direct responsibility for surgeons in the care of patients with potentially operative conditions.

\section{ACKNOWLEDGEMENTS}

Data for the study were provided by the Arkansas Clinical Data Repository (AR-CDR) maintained by the Department of Biomedical Informatics in the College of Medicine at the University of Arkansas for Medical Sciences (UAMS). The AR-CDR is approved to operate as an enterprise data resource to support research across UAMS. Data in the ARCDR comes from UAMS Electronic Medical Record (EMR), tumor registry, billing, and cancer genomic data and comprises encounters since 05/01/2014.

\section{CONFlicts OF INTEREST Disclosure}

The authors declare they have no conflicts of interest.
[2] Acker SN, Stovall RT, Moore EE, et al. Trauma remains a surgical disease from cradle to grave. The Journal of Trauma and Acute Care Surgery. 2014; 77(2): 219-225. PMid:25058245. https: //doi.org/10.1097/TA.0000000000000363

[3] Bilderback PA, Massman JD, 3rd, Smith RK, et al. Small Bowel 
Obstruction Is a Surgical Disease: Patients with Adhesive Small Bowel Obstruction Requiring Operation Have More Cost-Effective Care When Admitted to a Surgical Service. Journal of the American College of Surgeons. 2015; 221(1): 7-13. PMid:26095546. https://doi.org/10.1016/j.jamcollsurg. 2015.03.054

[4] Aquina CT, Becerra AZ, Probst CP, et al. Patients With Adhesive Small Bowel Obstruction Should Be Primarily Managed by a Surgical Team. Annals of Surgery. 2016; 264(3): 437-447. PMid:27433901. https://doi.org/10.1097/SLA.0000000000001861

[5] Daniak CN, Peretz D, Fine JM, et al. Factors associated with time to laparoscopic cholecystectomy for acute cholecystitis. World Journal of Gastroenterology. 2008; 14(7): 1084-1090. PMid:18286691. https://doi.org/10.3748/wjg.14.1084

[6] Moore EE, Maier RV, Hoyt DB, et al. Acute care surgery: Eraritjaritjaka. Journal of the American College of Surgeons. 2006; 202(4): 698-701. PMid:16571442. https://doi.org/10.1016/j.jamc ollsurg. 2005.12.007

[7] Qureshi A, Smith A, Wright F, et al. The impact of an acute care emergency surgical service on timely surgical decision-making and emergency department overcrowding. Journal of the American College of Surgeons. 2011; 213(2): 284-293. PMid:21601487. https://doi.org/10.1016/j.jamcollsurg. 2011.04.020

[8] Scherer LA, Battistella FD. Trauma and emergency surgery: an evolutionary direction for trauma surgeons. The Journal of Trauma. 2004; 56(1): 7-12. PMid:14749559. https://doi.org/10.1097/01.T A. $0000108633.77585 .3 B$

[9] Fingar KR, Stocks C, Weiss AJ, et al. Most Frequent Operating Room Procedures Performed in U.S. Hospitals, 2003-2012. 2014. Available from: https: //www.hcup-us . ahrq.gov/reports/statbriefs/sb18 6-Operating-Room-Procedures-United-States-2012.jsp

[10] Judkins SE, Moore EE, Witt JE, et al. Surgeons provide definitive care to patients with gallstone pancreatitis. American Journal of Surgery. 2011; 202(6): 673-677, discussion 677-678.
[11] Kulvatunyou N, Watt J, Friese RS, et al. Management of acute mild gallstone pancreatitis under acute care surgery: should patients be admitted to the surgery or medicine service? American Journal of Surgery. 2014; 208(6): 981-987, discussion 986-987.

[12] Lau B, Difronzo LA. An acute care surgery model improves timeliness of care and reduces hospital stay for patients with acute cholecystitis. The American Surgeon. 2011; 77(10): 1318-1321. PMid:22127078.

[13] Lehane CW, Jootun RN, Bennett M, et al. Does an acute care surgical model improve the management and outcome of acute cholecystitis? ANZ Journal of Surgery. 2010; 80(6): 438-442. PMid:20618197. https://doi.org/10.1111/j.1445-2197.2010.05312.x

[14] Michailidou M, Kulvatunyou N, Friese RS, et al. Time and cost analysis of gallbladder surgery under the acute care surgery model. The Journal of Trauma and Acute Care Surgery. 2014; 76(3): 710-714. PMid:24553538. https://doi.org/10.1097/TA.00000000000 00117

[15] Britt RC, Bouchard C, Weireter LJ, et al. Impact of acute care surgery on biliary disease. Journal of the American College of Surgeons. 2010; 210(5): 595-599, 599-601.

[16] LaFemina J, Sokal SM, Chang Y, et al. Effect of medical or surgical admission on outcome of patients with gallstone pancreatitis and common bile duct stones. Journal of Gastrointestinal Surgery: official journal of the Society for Surgery of the Alimentary Tract. 2008; 12(9): 1554-1560. PMid:18622658. https://doi.org/10.1007/ s11605-008-0580-1

[17] Moore BJ, White S, Washington R, et al. Identifying Increased Risk of Readmission and In-hospital Mortality Using Hospital Administrative Data: The AHRQ Elixhauser Comorbidity Index. Medical Care. 2017; 55(7): 698-705. PMid:28498196. https://doi.org/10.1 097/MLR.0000000000000735

[18] StataCorp. 2017. Stata Statistical Software: Release 15. College Station TSL. 